\title{
Geschichte im Fernsehen zwischen Faktualität und Fiktionalität: Das Dokudrama als Hybridform historischer Darstellung, mit einer Fallstudie zu Simon Schamas Rough Crossings
}

\author{
Barbara Korte
}

\section{Einleitung}

In der narratologischen Diskussion über faktuale bzw. „Wirklichkeitserzählungen" (Klein/Martinez 2009) hat die Historiographie besondere Aufmerksamkeit erfahren, nicht nur weil spätestens mit Hayden Whites Arbeiten (1973 und später) ihre literarische Dimension in den Blick gerückt ist. Im Fokus der Diskussion standen zumeist Beispiele mit ausgeprägter Literarizität und/oder kanonischem Status, ob im Genre des historischen Romans oder im Fall bedeutender Werke der Geschichtsschreibung. Weitaus weniger beachtet wurden bislang populäre Formate der Geschichtsdarstellung, die in Hinblick auf Interferenzen von Faktualität und Fiktionalität jedoch ergiebig sind. Fiktionalisierung, meist in Konjunktion mit einer ausgeprägten Narrativität, ist eines der Charakteristika populärer Geschichtsvermittlung, die seit ca. den 1990er Jahren in verschiedenen Medien vom historischen Krimi und Geschichtsfilm bis zu Computerspiel und Reenactment - eine Konjunktur erlebt. Ein Hauptanliegen populärer im Gegensatz zu akademischer oder schulischer Geschichtsdarbietung ist es, ein faktual belegtes Wissen über historische Welten in einer für die Rezipienten besonders ansprechenden und für die eigene Lebenswelt relevanten Form zu vermitteln:

In ihrer ästhetischen Gestaltung streben populäre Geschichtsdarstellungen eine hohe Allgemeinverständlichkeit und Zugänglichkeit an. Sie bieten ihren Rezipienten und Nutzern neben Information auch Unterhaltung (,Infotainment', ,Histotainment'), d. h. sinnliches Vergnügen, Entspannung und Spiel. Sie zeichnen sich durch sinnfällige, kohärente Narrative, durch Bebilderung und Formen der ,Belebung' aus [...]. Fakten und Imaginäres gehen Synthesen ein, die dazu beitragen, Geschichte spannend und für die Lebenswelt der Rezipienten anschlussfähig zu machen. Oft werden explizit „Brücken“ zwischen Gegenwart und Vergangenheit gebaut. Identifikationsangebote werden durch personalisierte und affektive Elemente unterstrichen. (Korte/Paletschek 2009: 14-15) ${ }^{1}$

Die verschiedenen Mediengattungen der populären Geschichtspräsentation figurieren historisches Material mit den ihnen jeweils zur Verfügung stehenden dis-

1 Die Forschergruppe „Historische Lebenswelten in populären Wissenskulturen“ (DFG FOR 875) an der Universität Freiburg (2007-13) hat populäre Geschichtskultur in diversen inhaltlichen und formalen Manifestationen untersucht. Für einen Überblick über Aktivitäten und Publikationen der Forschergruppe siehe http://portal.uni-freiburg.de/historische-lebens welten. 
kursiven Möglichkeiten. ${ }^{2}$ Im Folgenden wird von diesen Mediengattungen eine neuere Spielart der Geschichtsdokumentation im Fernsehen in den Blick genommen, nämlich das Dokudrama, in dem faktuales und fiktionales Erzählen eine besonders enge Beziehung eingehen, ${ }^{3}$ weil hier mehr oder weniger umfangreiche Teile der Dokumentation als dramatisierte und von professionellen Schauspielern gespielte Handlung präsentiert werden. Das Dokudrama ist also eine Hybridform und gerade als solche im Fernsehen des späten 20. und 21. Jahrhunderts beliebt. Es gibt weiterhin Dokumentarformate, in denen eine faktuale Darstellung dominiert und die vor allem mit Archivmaterial oder Zeitzeugeninterviews arbeiten; herausragende Beispiele sind die Dokumentationen von Ken Burns über den amerikanischen Bürgerkrieg (The Civil War, PBS, 1990) und den Zweiten Weltkrieg (The War, PBS, 2000). Aber Dokumentarformen, die punktuell oder fast gänzlich mit Spielszenen gestaltet sind, werden immer häufiger. Als Fallbeispiel mit einer besonders ausgeprägten Spielhandlung analysiert der zweite Teil dieses Beitrags das historische Dokudrama Rough Crossings von Simon Schama, in dem die fiktionalisierte Darstellung wesentlich dazu beiträgt, eine ,vergessene' Geschichte für Zuschauer wieder lebendig und für das Verständnis der Gegenwart relevant zu machen und so auch ein zentrales Ziel populärer Geschichtsdarstellung zu realisieren. Dieser Analyse vorangestellt sind kontextualisierende Anmerkungen zur Geschichtsdarbietung im Fernsehen und zur Gattung der Dokumentation.

\section{Geschichte im Fernsehen}

Die Vermittlung von Geschichte im Fernsehen verdeutlicht den Spagat populärer Geschichtsdarstellung zwischen faktenbasierter Wissensvermittlung und unter-

2 Zum Begriff der Mediengattung, der auf S. J. Schmidt zurückgeht, siehe Viehoff (2002, 126-27).

3 Für Eva Hohenberger besteht im Dokumentarfilm generell eine Tendenz, fiktionale Elemente zu inkorporieren, weil in ihrer Sicht jeder Dokumentarfilm zumindest minimal narrativ ist: „Auch wenn manche Theoretiker [...] den Dokumentarfilm generell als nichtnarrativ bezeichnen [...], ist heute unumstritten, daß Dokumentarfilme erzählen und daß sie sich dabei Verfahren bedienen, die der Spielfilm historisch bereitgestellt hat. Daß auch umgekehrt dokumentarische Authentisierungsstrategien in den Spielfilm Einzug gehalten haben, schwächt nicht nur die Gründungsmythen zweier strikt getrennter Gattungen zunehmend $\mathrm{ab}$ und gibt den Blick für deren Interferenzen frei, sondern deutet darauf hin, daß der Dokumentarfilm nicht als fiktionsfreie Diskursform zu begreifen ist" (Hohenberger 1998: 22-3). Da das Dokudrama die Narrativität der Dokumentation besonders ausbildet, trifft hier auch die folgende Beobachtung von François Jost in besonderem Maße zu: „Die Fiktion wird nur dann wirklich Teil des Dokumentarischen, wenn letzteres versucht, eine kohärente und logische Welt zu bilden: d. h. wenn alle Ereignisse zum Aufbau einer einzigen Bedeutung beitragen [...]. Also bewegt sich das Dokumentarische von dem Augenblick an langsam auf die Fiktion zu, in dem es sich den diegetischen Schemata, die in anderen fiktionalen literarischen oder filmischen Erzählungen bereits existieren, angleicht“ (Jost 1998: 225). Zu neueren Entwicklungen der Dokumentation im Fernsehen siehe Izod/Kilborn (2000), Kilborn/Izod (1997) und Kilborn (2003). 
haltsamer Präsentation geradezu exemplarisch, denn das Fernsehen, und zumal das öffentlich-rechtliche, ist noch immer ein Medium, das sich (auch) durch einen Bildungsauftrag definiert sieht. Historische und zeithistorische Stoffe können als ,reines 'Fernsehspiel inszeniert werden, das dem historischen Roman oder dem historischen Spielfilm insofern affin ist, als hier eine historische Welt durch Figuren erlebt und erfahren wird, und diese Figuren gänzlich erfunden sein können. Weitaus häufiger vermittelt das Fernsehen Geschichte aber zumindest partiell dokumentarisch, d. h. in Formaten, die mit ihrem spezifischen Diskurs deutlich signalisieren, dass sie Tatsachen präsentieren und sich mit ihnen auseinandersetzen.

Es ist behauptet worden, dass Dokumentarfilm und Geschichtsschreibung geradezu eine ,natürliche`Affinität aufweisen, und zwar nicht nur, weil der Film ein Gedächtnismedium ist, das einmal Archiviertes wieder zur Aufführung bringen und damit ,Geschichte' wieder vergegenwärtigen kann. Wie Eva Hohenberger und Judith Keilbach betonen, sind Dokumentarfilm und Geschichtsschreibung beide auch in besonderem Maße

der Faktizität der Welt verpflichtet. Sie interessieren sich für die Realität, d. h. für Ereignisse, die tatsächlich stattfinden oder stattgefunden haben, für deren Ursachen und Auswirkungen, ihren Ablauf oder die Erinnerungsspuren, die sie hinterlassen haben. Diese Verpflichtung auf die Realität findet ihren Ausdruck in einer Rhetorik des Faktischen, die sowohl die Geschichtswissenschaft als auch den Dokumentarfilm kennzeichnet. (2003: 8)

Neben einer „Rhetorik des Faktischen“ oder präziser einer faktualen Rhetorik, die die faktische Referenz des Dargestellten auf der Diskursebene affirmiert, ${ }^{4}$ bedarf die historische Fernsehdokumentation, wenn sie heute ein breites Publikum ansprechen will, offenbar aber auch eines hohen Anteils an fiktionalisierter Darstellung. Um Geschichte attraktiv, zugänglich und unterhaltsam zu präsentieren, ist nicht nur eine anschauliche Bebilderung nötig, die das Fernsehen exemplarisch liefern kann, sondern auch eine Erzählung, die über das emplotment hinaus Geschichte personalisiert und als Erfahrung individueller Menschen vermittelt. Letzteres wird durch typisch fiktionalisierende Elemente wie szenische Darstellung mit wörtlichen Dialogen oder durch Einblicke in Gedanken und Gefühle der dargestellten Personen erreicht, ${ }^{5}$ die im Fernsehen nicht nur verbal, sondern auch durch bild- und musikästhetische Effekte vermittelt werden. Die für heutige Sehgewohnheiten ansprechende historische Fernsehdokumentation verlangt also geradezu nach Interferenzen von Faktualität und Fiktionalität, wie sie das DokuDrama bereits in seiner Gattungsbezeichnung trägt.

Dabei kann der Schwerpunkt durchaus bei der fiktionalisierten Darstellung des ,Dramas' liegen, Faktualitätssignale dürfen aber nicht völlig ausgeblendet sein, weil ansonsten der pragmatische Faktualitäts-,Vertrag', der für die Rezeption

Zur Referenz auf Reales in den Medien siehe Segeberg (2009).

5 Siehe hier die Fiktionalitätssignale, die Dorrit Cohn (1990) anführt, sowie Monika Fluderniks Konzept der experientiality (1996). 
eines Textes oder Films als Tatsachendarstellung unabdingbar ist, nicht zustande kommt. Für den Zuschauer, der durch Geschichtssendungen nicht nur unterhalten, sondern auch informiert werden will, ist also ein Minimum an faktualer Rhetorik wichtig, und er wird ihm bereits durch die Gattungsbezeichnung DokuDrama paratextuell signalisiert. Wenn der Begriff Dokudrama, wie dies manchmal geschieht, auch für Geschichtsfilme mit rein fiktionaler Rhetorik verwendet wird, ${ }^{6}$ ist er zu unscharf und wird der besonderen Wirkung, die das Dokudrama (wie andere Hybridformen der Dokumentation ${ }^{7}$ ) ausübt, nicht gerecht. So schlägt auch Matthias Steinle aus dem deutschen Fernsehkontext heraus eine enge Definition des Dokudramas vor:

Kaum ein Konzept ist so schwer zu fassen wie das des ,Dokudramas' als Inbegriff des Hybriden aus fact und fiction. Während ,Doku' für das Versprechen von Information/Aufklärung, Bildung und Authentizität steht, verspricht ,Drama ' Unterhaltung, Schauspiel, Emotionen und sinnliche Attraktion. [...]

Im engsten Sinne der deutschen Fernsehgeschichte bezeichnet Dokudrama die von Heinrich Breloer zusammen mit Horst Königstein seit Anfang der 1980er Jahre entwickelte fernsehspezifische Form, dokumentarisches Archivmaterial mit Zeitzeugeninterviews und nachgestellten Spielszenen in einen sich gegenseitig ergänzenden und reflektierenden Zusammenhang zu setzen [...]. Nach einem ähnlichen Muster arbeitet für das ZDF das Team Guido Knopp und Hans-Christoph Blumenberg. [...] Vor allem bei den ZDF-Produktionen, aber auch in den jüngeren Breloer-Filmen, geht die Tendenz dahin, die verschiedenen Bildsorten nahtlos [...] in eine geschlossene Erzählung zu überführen. (2009: 149-50)

Das Fallbeispiel Rough Crossings wird zeigen, dass es neben den von Steinle genannten Optionen auch andere Möglichkeiten gibt, den Hybrideffekt des Dokudramas zu erzeugen.

Der Spagat zwischen Wissensvermittlung und Faktualitätsanspruch auf der einen Seite und fiktionalisiertem Spiel auf der anderen Seite stellt an den Dokumentarfilm allerdings auch schwierige Anforderungen, wie seine Theoretiker und Praktiker thematisiert haben. So bemerkt etwa Heinz-B. Heller:

Dokumentarfilm und Unterhaltung: Auf den ersten Blick scheint es wohl kaum ein unverträglicheres Begriffspaar zu geben. Die dem Dokumentarfilm (zumeist normativ) zugeschriebene Funktion, im Unterschied zu den erfundenen Wirklichkeiten des Spielfilms

6 Siehe allgemein zum Dokudrama auch Rosenthal (1999) und Paget (2011).

7 Siehe zu Intersektionen von Fakt und Fiktion in der Fernsehdokumentation auch Rhosed und Springer (2006). Abzuheben ist das Dokudrama u. a. von einer anderen neuen Dokumentarform, der Dokusoap, die sich von ,klassischen' Dokumentarformaten allerdings weit entfernt hat. Hierbei handelt es sich um eine Abwandlung von ,reality tv', das wirkliche Figuren in ungewöhnlichen Situationen ihres Lebens präsentiert, dabei in der Regel aber einem Script folgt. Historische Dokusoaps - wie der Prototyp 1900 House (Channel Four 2000) oder das deutsche Schwarzwaldhaus 1902 (SWR/ARD 2001/2) - versetzen Menschen aus ihrem Alltag in eine vergangene Welt und zeigen, wie sie sich in den Tücken und sozialen Rollen dieser Zeit zurechtfinden oder eben nicht. Dies hat Elemente der living histo$r y$, doch dominiert meist ein Schauwert über die Vermittlung von Tatsachenwissen über die Vergangenheit. Zum Wirklichkeitsfernsehen allgemein siehe Hill (2005). 
außerfilmische Realität faktengetreu und authentisch im Filmbild selbstevident zur Anschauung zu bringen, erscheint schwerlich vereinbar mit einem Rezeptionsversprechen oder einer -haltung, die - ungeachtet ihrer begrifflichen Unschärfen - im wesentlichen auf die Entfaltung von spielerischer Phantasie, sinnlichem Vergnügen und intellektuellem Lustgewinn setzt. Dies um so mehr, als sich in der Geschichte des Dokumentarfilms, mit dessen Anspruch auf die faktische Vergegenwärtigung außerfilmischer Wirklichkeiten, stets mehr oder weniger auch aufklärerische, wenn nicht gar didaktische Intentionen vermittelten; Zielvorstellungen, die dem Spielerischen der Unterhaltung, das immer auch Momente des Wirklichkeitsenthobenen impliziert, entgegen zu stehen scheinen. (1995: 97)

Ähnlich problematisiert Stefan Brauburger, Praktiker aus der ZDF-Redaktion Zeitgeschichte, die Entwicklungen bei der historischen Fernsehdokumentation:

Begriffe wie ,seriös‘, ,authentisch‘, ,glaubhaft‘, ,Tatsachen` oder ,echte Geschichten` werden nach wie vor mit der ,Dokumentation' assoziiert. Verändert haben sich jedoch die Erwartungen an Form und Gestaltung: Nähe und Spannung, nachgespielte Szenen auf hohem Niveau, aufwändige Grafiken und Animationen, tolle und beeindruckende Bilder, etwas fürs Auge, Einsatz von Musik, nicht zu viele Fakten, Emotionen, keine Belehrung und wenn möglich auch etwas Unterhaltung. Dieser Erwartungshorizont, der auch für zeitgeschichtliche Dokumentationen gilt, ragt unverkennbar in die Sphäre des Fiktionalen, zumindest was gestalterische Standards anbelangt. Tritt da womöglich das Faktische in den Hintergrund, wenn man den formalen Ansprüchen des Publikums genügen will? (2009: 203)

Für die Zwecke einer populären Darstellung werden die ,Kosten` der Fiktionalisierung aber in Kauf genommen, und dies nicht erst in jüngster Zeit. Die britische Fernsehproduzentin Taylor Downing schreibt über die erste große und maßstabsetzende Geschichtsdokumentation der BBC, die sich 1964 dem Ersten Weltkrieg widmete:

The narration was read with great solemnity by Sir Michael Redgrave. The series had a haunting musical accompaniment, and it was epic in its scope. It was a pioneering example of the effectiveness of combining archive film with eye-witness testimony, and in breaking the history of the war down into bite-sized chunks of narrative which are easily digestible in television time-spans. The Great War series was highly dramatic, and it was outstanding television. But it was not always good history, for its use of archive film was dubious to say the least. Feature film footage shot well after the war, often taken in film studios in the 1920s, was freely intercut with authentic record film.

But it was a great narrative, and it is narrative that is at the heart of the success of history on television. (2004: 9)

Neben einer starken Erzählung, dem Zwang zur attraktiven Bebilderung (die auch heute oft noch zur unkommentierten Verwendung von Spielfilmmaterial anstelle von authentischem historischen Filmmaterial führt) sowie einer Emotionalisierung des historischen Materials u. a. durch die Begleitmusik benennt Downing Darstellungsmittel, die sich mittlerweile als Konventionen der Geschichtsdokumentation im Fernsehen etabliert haben und als Faktualitätssignale für das Publikum fungieren können: der als Voice-Over aus dem Off gesprochene Kommentar und, sofern vorhanden, eingeschnittene Augenzeugen-Interviews 
und die Verwendung von filmischem oder fotografischem Archivmaterial bzw., wo solches nicht vorliegt, anderer Zeitdokumente und Artefakte.

Ein kurzer Exkurs in die Theorie des Dokumentarfilms kann das Verständnis für die faktuale Rhetorik der Geschichtsdokumentation noch weiter schärfen, denn in der Diskussion über den Dokumentarfilm ist nicht nur der Aspekt seiner Referenz auf eine tatsächliche ,Wirklichkeit ${ }^{6}$ zentral, ${ }^{8}$ sondern immer auch die Tatsache, dass daneben über den Diskurs ein Faktizitätseindruck hervorgerufen werden muss. Dass Referenz und Diskurs dabei potenziell voneinander unabhängig sind, zeigt sich darin, dass Dokumentarfilme nicht nur von jeher nachgestellte Szenen enthalten haben (und die Digitalisierung dafür heute völlig neue Möglichkeiten bietet), ${ }^{9}$ sondern dass es auch ,falsche ${ }^{6}$ Dokumentarfilme gibt, in der durch Darstellungskonventionen eine echte Referenz nur vorgetäuscht wird. ${ }^{10}$ Das Dokumentarische des Dokumentarfilms ist nicht aus der Ontologie seiner photographischen Bilder ableitbar, ${ }^{11}$ sondern die Rezeption eines Films als Dokumentarfilm ist dadurch bestimmt, dass die Zuschauer annehmen, dass das im Bild Gezeigte wirklich ist oder war, und zwar aufgrund ihres Weltwissens, paratextueller Signale (neben Gattungsbezeichnungen im Fernsehen z. B. auch bestimmte Sender und Programmplätze), Hinweise und eben bestimmten rhetorischen Konventionen der Gattung Dokumentarfilm: „The sense that a film is a documentary lies in the mind of the beholder as much as it lies in the film's context or structure".

So fasst Bill Nichols $(2001,35)$ den Umstand, der oben bereits mit der Vorstellung eines Faktualitätsvertrags eingeführt wurde. ${ }^{12}$ Nichols präzisiert dies mit einem Vergleich der Rezeptionshaltungen von Dokumentar- und Spielfilm:

8 Schon die frühen Theoretiker des Dokumentarfilms, die gleichzeitig namhafte Praktiker waren wie Vertov in Russland und Grierson in Großbritannien (der den Begriff documenta$r y$ erstmals 1926 in seiner Rezension von Robert Flahertys Film Moana verwendete), lokalisierten das Wesen des Dokumentarfilms in seiner besonderen Beziehung zur Wirklichkeit; siehe die Texte in Hohenberger (1998).

9 Zum Beispiel musste eine der berühmtesten Szenen aus The Battle of the Somme (1916), einem britischen Dokumentarfilm über den Ersten Weltkrieg, die in fast allen späteren Dokumentationen über diesen Krieg gezeigt wird, nachgestellt werden, weil weder der Kameramann noch die Kamera das Filmen eines echten ,going over the top' der Soldaten überlebt hätten. Schauplatz und Akteure waren allerdings authentisch. Zur Häufigkeit von Nachstellungen und Fälschungen in frühen Dokumentarfilmen siehe auch Barnouw (1983: 25).

10 Zum sog. pseudo-documentary, mockumentary oder fake documentary siehe Hattendorf (1995), Roscoe und Haight (2001), Juhasz und Lerner (2006) sowie Bayer (2008).

11 Vgl. hier auch Hohenberger: „Obsolet scheinen alle Ansätze, die aus der Aufzeichnung von Realität eine spezifische Qualität des Dokumentarfilms gewinnen wollen. [...] Die eindeutigen Zuordnungen von Original (Welt) und Bild brechen zudem mit den technologischen Entwicklungen der digitalen Bilderproduktion auf“ (Hohenberger 1998: 28).

12 Auch Eva Hohenberger nimmt einen solchen pragmatischen Vertrag an: „Prämisse eines pragmatischen Zugangs zum Dokumentarfilm ist, daß er sich eben nicht anhand textueller Verfahren, seien sie nun narrativ oder nicht, vom Spielfilm unterscheiden läßt, sondern daß er sich gleichsam qua Vertrag zwischen Zuschauern und Text erst konstituiert. Ohne Weltwissen einerseits und ohne Wissen um filmische (Genre-)Konventionen andererseits kann zwischen Spiel- und Dokumentarfilmen nicht unterschieden werden, erst recht nicht dort, 
In fiction [...] we turn our attention from the documentation of real actors to the fabrication of imaginary characters. We suspend our disbelief in the fictional world that opens up before us. In documentary, we remain attentive to the documentation of what comes before the camera. We uphold our belief in the authenticity of the historical world represented on screen. We continue to assume that the indexical linkage of sound and image to what it records attests to the film's engagement with a world that is not entirely of its own design. Documentary re-presents the historical world by making an indexical record of it; it represents the historical world by shaping this record from a distinct perspective or point of view. The evidence of the re-presentation supports the argument or perspective of the representation. (Nichols 2001: 36-7)

Dokumentarfilme sind deshalb selbst keine Dokumente, basieren aber auf der dokumentartigen Qualität ihrer Komponenten:

Documentaries are not documents in the strict sense of the word, but they are based on the document-like quality of elements within them. As an audience we expect to be able both to trust to the indexical linkage between what we see and what occurred before the camera and to assess the poetic or rhetorical transformation of this linkage into a commentary or perspective on the world we occupy. We anticipate an oscillation between the recognition of historical reality and the recognition of a representation about it. This expectation distinguishes our involvement with documentary from our involvement with other film genres. (Nichols 2001: 38-9)

Nichols' Hinweis, dass der Dokumentarfilm einerseits Re-Präsentation (von etwas Existierendem oder Gewesenem) ist, andererseits aber auch eine in bestimmter Weise figurierte Repräsentation, betont den Umstand, dass Dokumentarfilme mit bestimmten Darstellungsabsichten gemacht werden und entsprechend auf ihre Darstellungsrhetorik oder -poetik hin analysiert werden können. Nichols nennt hier für den Dokumentarfilm Ausdruckselemente, die zum Teil bereits oben in Hinblick auf die Rhetorik historischer Fernsehdokumentationen erwähnt wurden und die alle dazu beitragen, in Zuschauern einen Faktualitätseindruck hervorzurufen oder zu stärken:

[T] he use of Voice-of-God commentary, interviews, location sound recording, cutaways from a given scene to provide images that illustrate or complicate a point made within the scene, and a reliance on social actors, of people in their everyday roles and activities, as the central characters of the film are among those [elements] that are common to many documentaries. (Nichols 2001: 26)

Gemäß ihrer Aussageabsichten folgen Dokumentarfilme einer bestimmten Logik, die die Argumentation entwickelt und dafür bestimmte Behauptungen oder Tatsachen akzentuiert, während Spielfilme auf die Entwicklung einer Handlung und/oder eines Charakters angelegt sind. So wollen Dokumentarfilme zum Beispiel die Verantwortlichkeit von Kriegsparteien für den Ausbruch eines Konflikts klären, geheime Pläne aufdecken, die Chronologie von Ereignissen verdeutlichen oder die Persönlichkeit eines historischen Akteurs erklären. Unterschiedliche Lo-

wo auch Dokumentarfilme auf einem Plot beruhen, die Handlung ihrer Helden psychologisch motivieren und ein raum-zeitliches Kontinuum schaffen" (Hohenberger 1998: 25). 
giken gehen mit unterschiedlichen Darstellungsstrategien einher, wie etwa bei der Handhabung des Bildschnitts. Typisch für den Spielfilm ist das continuity editing, d. h. der scheinbar unsichtbare Schnitt, der den Fluss der Handlung und die Illusion der Zuschauer nicht stört. Im (reinen) Dokumentarfilm ist diese Art des Schnitts von untergeordneter Bedeutung, während verdeutlichende oder kommentierende Montagen, die Beziehungen herstellen, für die Argumententwicklung eine wichtige Funktion haben: „things share relationships in time and space not because of the editing but because of their actual, historical linkages. Editing in documentary often seeks to demonstrate these linkages" (Nichols 2001: 27).

Dokumentarfilme unterscheiden sich laut Nichols schließlich durch Präferenzen für bestimmte Aussagemodi, die sie dominant oder auch in Kombination einsetzen. Der (1) expositorische Modus, d. h. die Erklärung, ist für den Dokumentarfilm prägend und manifestiert sich vor allem im verbalen Kommentar, während der (2) beobachtende Modus Kommentare bewusst zurückhält. In einem (3) ,teilnehmenden' Modus (participatory mode) lässt der Dokumentarfilmer hingegen seine Interaktion mit dem Sujet erkennen, die sich bis zum (4) performativen Modus steigern kann, wenn die subjektiven und expressiven Seiten dieses Engagements hervorgehoben werden. Der (5) reflexive Modus lenkt die Aufmerksamkeit auf die Darstellungskonventionen des Dokumentarfilms selbst und stellt ein Meta-Element dar, während der (6) poetische Modus ästhetische Elemente der Darstellung selbst (visuelle Assoziationen, tonale und rhythmische Qualitäten etc.) in den Vordergrund rückt (siehe Nichols 2001: 33-4).

In ihrer Doku-Komponente verwenden historische Dokudramen zahlreiche Elemente und Konventionen des klassischen Dokumentarfilms und seiner faktualen Rhetorik. Daneben tritt aber, wie schon gezeigt, in erheblichem Maße eine ,dramatische' Komponente, welche die Geschichte fiktionalisiert. Das $\mathrm{Zu}$ sammenspiel dieser unterschiedlichen Rhetoriken wird nun im Folgenden für Simon Schamas Rough Crossings betrachtet, ein Dokudrama, das seinen Status als Dokudrama vor allem der starken Präsenz seines Autor-Erzählers Simon Schama verdankt. Nicht nur spricht Schama den Kommentar selbst, sondern er tritt auch vor die Kamera und addressiert sein Publikum direkt. Von Nichols' dokumentarfilmischen Modi sind dabei, wie sich zeigen wird, der expositorische, der partizipatorisch-performative und der reflexive dominant, und an einigen herausgehobenen Stellen wie dem Schluss hat die dokumentarische Rhetorik auch dezidiert ,poetische $e^{6}$ Momente. Die prägnante und durch Schama personalisierte faktuale Rhetorik sorgt dafür, dass die dokumentarische Ebene von Rough Crossings sich gegenüber der stark ausgeprägten Spielhandlung behaupten kann.

\section{Fallstudie: Rough Crossings}

Simon Schama ist Kunsthistoriker und Historiker an der Columbia University in New York und ein Star unter den britischen Fernsehhistorikern, der für seine le- 
bendige und eindringliche Geschichtsvermittlung geschätzt wird. Einem nichtakademischen Publikum wurde Schama vor allem durch seine 15-teilige History of Britain (BBC 2000-2002) bekannt. Die Popularität dieser Serie machte es möglich, dass Schama auch sein Rough Crossings-Projekt (BBC 2, 2007, Autor Simon Schama, Regie Steven Condie) realisieren konnte, obwohl er hier eine unbekannte Geschichte erzählt, die sich in groben Zügen wie folgt skizzieren lässt.

1792 wanderte eine Gruppe ehemaliger amerikanischer Sklaven vom heute kanadischen Nova Scotia nach Sierra Leone in Westafrika aus. Es handelte sich um Menschen, die im amerikanischen Unabhängigkeitskrieg auf Seiten der Briten gekämpft hatten, um der Sklaverei zu entkommen. Ihnen wurde die Freiheit versprochen, und nach dem Krieg wurden sie von den Briten in dem ihnen verbliebenen Teil Nordamerikas angesiedelt. Das Leben in Neuschottland erwies sich allerdings kaum besser als die Sklaverei. Als die Freigelassenen von der Absicht britischer Abolitionisten hörten, in Freetown (Sierra Leone) eine ,Provinz der Freiheit' zu gründen und damit Pläne für eine Kolonie freigelassener Sklaven in Afrika wieder aufleben zu lassen, meldeten sie sich für das Projekt und entsandten ihren Anführer Thomas Peters nach London, um die Bedingungen auszuhandeln. Das Umsiedlungsprojekt kam zustande, allerdings nicht unter der Führung von Peters, sondern der eines weißen Briten. John Clarkson, ein Bruder des prominenten Abolitionisten Thomas Clarkson, organisierte den "Exodus" nach Freetown und machte sich die Sache der früheren Sklaven zu eigen. Nach der rauen und beschwerlichen Überquerung des Atlantiks, bei der Clarkson fast an einem Fieber starb, erwarteten die Auswanderer jedoch neue Enttäuschungen, und sie fühlten sich wiederum von den Briten betrogen. Statt sich in Freetown wie versprochen selbst regieren zu können, wurde ihnen Clarkson - auch zu seiner eigenen Überraschung - als Gouverneur einer nun strikt auf Gewinn hin angelegten Handelskolonie vorgesetzt. Mit Mühe gelang es Clarkson, die Grundlagen einer friedlichen Gesellschaft zu legen, aber er wurde dann nach England zurückgerufen und von seiner Aufgabe als Gouverneur entbunden. Die Kolonie in Freetown verfiel danach in Unfreiheit und Gewalt.

Vor seinem Dokudrama hatte Schama diese Geschichte bereits in einem umfangreichen historiographischen Werk verarbeitet, das, ebenfalls unter dem Titel Rough Crossings, 2005 erschien und in der für Schama typischen experimentellen Erzählweise mit internen Fokalisierungen der historischen Protagonisten arbeitet. Das 90-minütige Dokudrama war für ein breiteres Publikum und als FernsehEvent konzipiert. Es wurde zur besten Abendsendezeit am 23. März 2007 ausgestrahlt, ${ }^{13}$ anlässlich eines in Großbritannien damals weit beachteten Jahrestages, nämlich dem der Abschaffung des Sklavenhandels durch das britische Parlament

13 In den USA wurde ein anderer, passender Kontext für die Erstausstrahlung gefunden, nämlich der Black History Month im Februar 2009; das Dokudrama wurde von einer amerikanischen Firma ko-produziert. 
im Jahr 1807. ${ }^{14}$ Dies verschaffte dem Dokudrama eine aktuelle Relevanz im Kontext einer allgemeinen gesellschaftlichen Diskussion über eine neue Geschichtskultur für das multiethnische Britannien im 21. Jahrhundert. Diese Relevanz trug dazu bei, die Unbekanntheit der in Rough Crossings präsentierten Geschichte und ihrer Protagonisten zu kompensieren. Ein anderes Manko dieser Geschichte für das Fernsehen bestand in der Tatsache, dass Schamas Quellen aus dem späten 18. Jahrhundert kaum Material für eine attraktive ,authentische' Bebilderung boten. Insofern war ein Dokudrama, das seine historische Erzählung überwiegend in fiktionalisierter Spielhandlung und mit starker Erzählerpräsenz vermittelt, ein ideales Format für Schamas Anliegen, eine vergessene Geschichte wieder lebendig und ihre Bedeutung für die Gegenwart wieder präsent zu machen. Die Quellen, die Schama für seine historischen Recherchen verwendete (wie Urkunden, Briefe und John Clarksons Tagebuch) und die er in seinem Buch Rough Crossings ausführlich zitiert, werden im Dokudrama vollständig integriert und so als ,Quellen kaschiert: einerseits erscheinen sie als Teil von Schamas Kommentartext, andererseits werden sie in der Spielhandlung von den Figuren gesprochen, entweder im Dialog oder in Voice-Overs, die ihre Gedanken vermitteln.

Aufgrund der prominenten dramatischen Inszenierung der historischen Handlung bestehen die Faktualitätssignale in Rough Crossings, mit Ausnahme eines Zwischentitels (s. u.) und der Originalschauplätze in Sierra Leone, New York und London, die die Zuschauer aufgrund ihres Weltwissens als, echt' identifizieren können, vor allem in der Inszenierung Schamas als Autor-Erzähler und Kommentator, wobei allerdings dieser Marker durch die Präsenz und Prominenz Schamas besonderes Gewicht erhält. ${ }^{15}$ Wie schon gesagt, ist der Star-Historiker nicht nur zu hören, sondern immer wieder auch zu sehen, und zwar explizit in der Rolle des Historikers, der die Geschichte nicht nur mit Autorität erzählt, sondern auch ihre Bedeutung erklärt. Dabei hält sich Schama mit Wertungen der historischen Ereignisse und ihrer Protagonisten nicht zurück und legt sein persönliches Interesse an der Geschichte immer wieder offen. Die ,Logik', die Rough Crossings als große Argumentationslinie zugrunde liegt, ist die Feststellung, dass die hier - von Schama - wieder zutage geförderte Geschichte den Menschen heute etwas zu sagen hat, und dies affirmiert nicht nur Schamas Kommentar, ${ }^{16}$ sondern auch die visuelle Inszenierung: die Bilder, die zum Kommentar zu sehen sind, und ein Schnitt, der Gegenwart und Vergangenheit systematisch verknüpft.

14 Vgl. zur Einbindung von Rough Crossings in das geschichtskulturelle Umfeld dieses Gedenktages auch Korte/Pirker (2011: 81-98).

15 Siehe in diesem Kontext auch François Jost zur Bedeutung der Erzählstimme im Dokumentarfilm: „In der Konstruktion einer diegetischen Welt oder in der Konstruktion eines Universums des argumentativen Diskurses ist es nachweisbar, daß die Stimme und der Kommentar eine dominierende Rolle spielen. In einem Versuch, die ,Stufen von Dokumentarität' ${ }^{`}$ zu erklären, ist es daher wichtig, die Beziehung zwischen der Stimme und dem, was der Zuschauer tatsächlich sieht, in Betracht zu ziehen“ (1998: 226).

16 Zur Selbstinszenierung von Historikern siehe auch Jaeger (2009). 
Dass Schama als Vermittler persönlich im Bild auftritt, trägt auch dazu bei, den Mangel seines dokumentarischen Materials an historisch authentischen Bildern, O-Tönen und lebenden Augenzeugen auszugleichen, so dass die Visualisierung des Dokudramas nicht auf die fiktionalisierte Spielhandlung, das reenactment mit professionellen Schaupielern, beschränkt ist.

Reenactments in der historischen Fernsehdokumentation sind unter Historikern nicht unumstritten, von Fernsehpraktikern jedoch akzeptiert. So stellt Erwin Leiser fest: „Wenn das historische Filmmaterial nur ein kollektives Geschehen beinhaltet, muß es durch Zitate aus Spielfilmen oder durch Spielszenen ergänzt werden, die mit Schauspielern neu zu drehen sind“ (1992: 43). Noch dezidierter rechtfertigt Taylor Downing die Praxis der Nachstellung historischer Ereignisse, gerade für Epochen, für die es keine audiovisuelle Aufzeichnung gibt:

For me, historical re-enactments on television are perfectly justifiable when they evoke the spirit of the moment, the detail of a description, or the nature of some machine or piece of technology. [...]

[...] re-enactments are justifiable in allowing the viewer to see something that a cameraman would have shot had the technology of the time allowed it. [...] reconstructions can take us to places that no movie cameras ever went near. [...]

Television can now evoke periods of history outside the twentieth century, and we can explore topics that no one bothered to film or was able to film at the time. [...] The use of reconstructions has been criticised on the grounds that history is concerned with reality and evidence, whereas reconstructions are about ,pretend' and about fantasy, and are essentially phoney. [...] But as a television producer I operate in a competitive market, and reconstructions do attract viewers, for the very good reason that they bring history alive that would otherwise be dead. (2004: 13 und 14)

Das Reenactment in Rough Crossings hat eine hochwertige Spielfilmqualität und lädt die Zuschauer zur Empathie mit den dargestellten Figuren ein. Aber auch die Erzähl- und Kommentarebene ist aufwändig inszeniert und in ihren Effekten auf die Zuschauer genau kalkuliert. Dies wird bereits in der Anfangssequenz des Dokudramas deutlich, in der die Zuschauer nicht nur an die vergessene Geschichte der Ex-Sklaven aus Nova Scotia herangeführt werden, sondern auch an die Art und Weise, in der das Dokudrama seine fiktionalisierte Spielhandlung (die Vergangenheitsebene) und seine faktualisierende Erzählebene beständig mischt und miteinander verflicht: Noch vor der Titelsequenz beginnt Rough Crossings, für die Zuschauer zunächst irritierend und so Aufmerksamkeit weckend, mit einer Folge sehr kurzer, fetzen- und fast geisterhafter Einstellungen, die afrikanische Gesichter zeigen. Aufgrund der Kostümierung lassen sie sich sicher der Vergangenheit zuordnen. Darüber sind Satzfetzen zu hören: „It's daybreak! It's daybreak! Take us to your Province of Freedom... of the world... Where we will... Hallelujah! Freedom!“. Danach sind Bilder von Menschen auf einem Segelschiff zu sehen; der Kommentar Schamas setzt ein und kündigt eine Geschichte an, die wiederentdeckt werden muss, weil sie für die Gegenwart signifikant ist:

There are some stories so big, stories that tell us who we are, what we've become, you can't believe they ever got away, vanished from the history books. [Im Bild sind wieder 
Gesichter, aber jetzt nicht nur aus der Vergangenheit, sondern auch aus der Gegenwart; die beiden Zeitebenen wechseln sich dabei ab. Schama kommt in Großaufnabme ins Bild:] But then, I had no clue about this story myself. [Über den folgenden Sätzen sind Bilder der entflohenen Sklaven zu sehen, die im Unabbängigkeitskrieg kämpfen:] Escaped American slaves who fought for King George, not George Washington, in the American Revolutionary War, who flocked to the British flag, thousands of them. And who believed so badly in British freedom, they went through every kind of hell to get it. [Bilder von Schama in einer Straßenszene in Afrika.] A few years ago, though, I found that story, or rather it found me. [Die Bilder zeigen wieder die Vergangenheitshandlung und ihre wichtigsten Protagonisten:] A story of three men, two African-Americans, one young white Englishman. A soldier, a preacher, a sailor. And they have haunted me ever since. And so they should, those ghosts, black and white, tugging at our memory. You want to know what it means to be British, American, African now? [Die Bilder zeigen eine urbane Szene der Gegenwart.] What it means to be enslaved? What it means to be free? Then we need this story. We owe our ancestors this moment. [Es folgen Bilder aus Vergangenheit und Gegenwart, bis der Titel Rough Crossings erscheint.] (00:00-01:48) ${ }^{17}$

Die Sequenz folgt der von Nichols beschriebenen Tendenz, dass im Dokumentarfilm der Schnitt nicht unsichtbar ist, sondern explizit eingesetzt wird, um inhaltliche oder argumentative Bezüge zu illustrieren - in diesem Fall die Schama wichtigen Beziehungen zwischen Vergangenheit und Gegenwart. Die Fragmentarität der schnell geschnittenen Bilder und die Knappheit der ersten Informationen sind darauf angelegt, das Interesse der Zuschauer zu vertiefen und Emotionen zu wecken. Darüber hinaus suggerieren die Bild- und Sprachfetzen, dass im Folgenden eine Geschichte erzählt wird, deren Fragmente durch den Historiker erst wieder gefunden und zu einer Erzählung zusammengesetzt werden mussten.

Gleich nach dem Titel „Rough Crossings“ folgt ein Zwischentitel, der sich bereits mit seiner Schriftlichkeit von der vorangegangenen Darstellung abhebt und ein sehr deutliches Faktualitätssignal setzt, indem er die Wahrheit der darzustellenden Geschichte bezeugt und Schamas Quellenmaterial benennt: „This is a true story. The events are based on journals, letters \& historical records“ (01:5501:58). Unmittelbar im Anschluss setzen Bilder eines Feuerwerks ein, dessen Ort und Zeit ein Untertitel identifiziert: „New York. July 4th 2006“. Die Unabhängigkeit wird in den USA heute gefeiert, aber, so spricht Schamas Kommentar zu den Bildern aus, den Sklaven, von denen seine Geschichte erzählt, wurde Freiheit noch verweigert. Hier wird eine Behauptung angelegt, die Schama am Ende des Dokudramas explizit ausformulieren wird: Die Ereignisse, die Rough Crossings erzählt, illustrieren, dass die Protagonisten dieser Geschichte einen Beitrag dazu leisteten, dass die Sklaverei letztendlich überwunden werden konnte. Im Verlauf des Dokudramas sieht man Schama immer wieder in Straßenszenen in New York, London und in Sierra Leone, die andeuten, was heute aus dem ehemaligen transatlantischen Dreieck des Sklavenhandels geworden ist. Sie unterstreichen Schamas Behauptung, die von ihm erzählte Geschichte sei relevant für das Verständnis unserer heutigen Welt. Im Anschluss an die Anfangssequenzen wechseln

17 Transkribiert wird die im Literaturverzeichnis angegebene DVD. 
sich Gegenwart (dokumentarische Bilder) und Vergangenheit (Spielfilmbilder) zunächst weiter ab; dabei führt Schamas Kommentar tiefer in seine Geschichte ein, nicht ohne Ironie gegenüber den gottesfürchtigen britischen Abolitionisten, mit deren Billigung die geplante ,Province of Freedom' schließlich doch wieder zu einer auf Profit ausgerichteten Kolonie wurde.

Das Drama der gespielten Teile von Rough Crossings entfaltet sich nicht nur über den Plot, sondern sehr stark auch über die Zeichnung der historischen Figuren. Sie sind dank Fiktionalisierung runde Charaktere, an deren Wahrnehmungen, Erinnerungen und Gefühlen die Zuschauer teilnehmen können. Besonders ausgeprägt ist dies in der Darstellung von John Clarkson, der im Verlauf der Handlung einen Lern- und Reifungsprozess durchmacht und vom jungen Marineoffizier zu einer verantwortlichen Führungspersönlichkeit wird. In Nova Scotia erfährt Clarkson, auch stellvertretend für die Zuschauer, eine Initiation in das Schicksal der früheren Slaven, für die er zunehmend Mitgefühl empfindet. Deren traumatische Vergangenheit wiederum wird nicht nur erzählt, sondern in kurzen Flashbacks auch im Bild inszeniert. So erinnert sich der Baptistenprediger David George, der neben Peters eine Leitfigur der Auswanderer ist und den Glauben an Gerechtigkeit hochhält, wie seine Mutter zu Tode gepeitscht wurde (23:46-24:22). Die Charaktere des Dokudramas haben also eine Interiorität, an der die Zuschauer teilhaben können, und diese Interiorität wird auch durch die Mimik der Figuren vermittelt, die oft in Nah- und Großaufnahmen gezeigt werden. Die Figurenrelationen werden ebenfalls dramatisch gestaltet, besonders der Antagonismus zwischen Clarkson und Peters. Peters fühlt sich hinter dem weißen Engländer zurückgesetzt und beklagt sich darüber, dass man nicht ihm das Oberkommando über die Auswanderungsschiffe gegeben hat:

[Peters:] My opinions. Your decisions [lacht verächtlich], Commodore Clarkson. [Clarkson:] There can only be one man in charge, Peters. [Peters blickt auf das Meer und sagt:] You know what's at the bottom of that ocean? A trail of bones that leads from here to Africa. [Clarkson geht weg, obne zu antworten]. (32:48-33:18)

Die fiktionale Rhetorik dieser Szene wird ergänzt durch einen Kommentar Schamas, der, direkt an die Zuschauer gesprochen, die Enttäuschung von Peters nachvollzieht:

It was bound to happen, this falling out, wasn't it? For Peters, this young Englishman was just a functionary sent by men in London to organise the sailing. If it was to truly be a voyage to liberty, then he, Peters, who had shed his blood for it, was the natural leader of its self-determination. (33:36-33:54)

Nach der Atlantiküberquerung zeigt die Spielhandlung fast unmittelbar die Enttäuschung aller Hoffnungen in Freetown. Die Überfahrt, die für die ehemaligen Sklaven eine Umkehr der berüchtigten Middle Passage von Afrika in die Sklaverei sein sollte, führt sie in erneute Not und Abhängigkeit, da die Sierra Leone Company ihre Zusage, Hütten und Lagerhäuser bereitzustellen, nicht eingehalten hat. Hier, zu Beginn eines neuen Handlungsabschnitts, tritt auch Schama wieder pro- 
minent ins Bild, um die Enttäuschung zu kommentieren. Gleich danach stellt eine erneute Montage von fiktionalisierter Vergangenheit und dokumentarischer Gegenwart einen Bezug zwischen den Ex-Sklaven in Freetown und den heutigen Bewohnern Sierra Leones her, die der Kommentar aber nicht weiter erläutert und die die Zuschauer offensichtlich irritieren und zum Nachdenken bringen soll. Das verunsichernde Moment der Montage ist besonders stark, als eine Straßenszene der Gegenwart mit dem nächsten Bild unmittelbar in die Vergangenheitshandlung übergeht (50:13-50:23). Die Technik ähnelt dem ,unsichtbaren' Schnitt im Spielfilm, hat hier aber eine störende Funktion, da Grenzlinien der Zeit und zwischen faktualer und fiktionalisierender Darstellung überschritten werden.

Nachdem der Traum der ,Province of Freedom' sein unrühmliches Ende genommen hat, inszeniert sich Schama im Schlussteil von Rough Crossings (ab 1:21:00) noch einmal prominent in der Rolle des Historikers, der den Zuschauern als Autorität die Relevanz der jetzt fertig erzählten und dem Vergessen entrissenen Geschichte noch einmal abschließend - und fast überdidaktisch - erklärt. Begleitend zu dem folgenden langen Kommentar, der auch metahistorische Reflexionen enthält, sind zunächst Bilder der Armut im heutigen Sierra Leone zu sehen, die am Ende aber hoffnungsvolleren Bildern eines Jungen in einer Schule weichen. Dazwischen ist Schama zu sehen, der, ebenfalls in Afrika, die Zuschauer direkt anspricht:

Well, history isn't Hollywood. In history, feel-good endings are hard to come by. This great experiment in black democracy did fall apart, but the idea of Freetown endured. After the abolition of slavery, Sierra Leone became the country to which those liberated from the slave forts and ships and plantations flocked for a new life as free Africans. But, of course, peace and freedom are always fragile victories. You don't need me to tell you that; of late, Sierra Leone has had more than its fair share of war, sorrow and slaughter. And that's why we need to cherish the story of Peters and Clarkson and George. The precious moment when determined men and women, black and white, endured the worst to bring about a modestly good beginning. A small, decisive turn to the light. That's all we can ever ask from history really, a memory of free will exercised against the odds. So remember this part of your African, American and British history. Try and imagine what those most unlike you went through. [Bilder, in denen Gegenwart und Vergangenheit wieder durchmischt sind.] And then take that murmur of the past, that needful little haunting, into our shared, uncertain future. [Man hört Stimmen und Gesänge aus der Vergangenheit.]

Die zentrale Behauptung, dass man sich vom ,Gemurmel der Vergangenheit' verfolgen lassen soll, wird in den letzten Einstellungen des Dokudramas (ab 1:23:25) noch einmal pointiert inszeniert: In stimmungsvollen, poetischen Bildern ist Schama in der Abendsonne am Strand in Afrika zu sehen, und nach getaner Arbeit gönnt sich der Historiker ein Bier. Neben ihm steht ein Kofferradio, das in Großaufnahme gezeigt wird, um deutlich zu machen, dass die Stimme aus der Vergangenheit, die man hört, nämlich die des Predigers George, aus seinem Lautsprecher kommt; ganz wörtlich ist die Vergangenheit somit ein Teil der Gegenwart geworden und spricht zu Schama und gleichzeitig zu den Zuschauern. Dabei formuliert George ein hoffnungsvolles Gebet, das, wie ein Schnitt in die Spielhandlung zeigt, 
an Kinder der Freetown-Kolonie gerichtet war. Ein letztes Mal arbeitet das Dokudrama also mit seinem zentralen rhetorischen Mittel, nämlich dem Wechsel der Darstellungsebenen, die hier programmatisch ineinander fließen:

[George aus dem Lautsprecher:] Should any person have a wicked thought in his heart [George in der Spielhandlung zu den Kindern:] to disturb the peace and comfort of the colony, let him be rooted out, O God, from the face of the earth. [Schnitt zu Schama neben dem Radio, aus der Georges Stimme weeiter tönt:] May this colony, O Lord, live in unity and godly love. [Schnitt zu George in der Spielhandlung:] Now and forever. Amen. [Schnitt zu Bildern von einem Kind am Strand von Sierra Leone heute und Abspann.]

Mit ihrem Spiel mit Darstellungsebenen bildet diese Schlusssequenz von Rough Crossings nicht zuletzt einen meta-generischen Kommentar über das Dokudrama und seine hybride Darstellungsstrategie. Einerseits existiert die Präsentation der erzählten Geschichte als faktenbasierte Spielhandlung, deren - im Fall von Rough Crossings durchgängige - Fiktionalisierung wesentlich dazu beiträgt, eine den $\mathrm{Zu}-$ schauern unbekannte Geschichte zu verlebendigen, zu personalisieren und emotional erfahrbar zu machen. Andererseits gibt es eine Kommentarebene mit stark präsentem Autor-Erzähler, von der deutliche Faktualitätssignale ausgehen; diese wird aber auch dazu genutzt, eine persuasive (siehe Lipkin 2002) und didaktische Funktion zu erfüllen und den (direkt angesprochenen) Zuschauern die Relevanz des durch das Dokudrama vermittelten historischen Wissens für ihre eigene Welt zu verdeutlichen. Schamas Dokudrama realisiert so eine der zentralen Wirkungsabsichten, die Bill Nichols für den Dokumentarfilm beschreibt: „Documentary film and video stimulates epistephilia (a desire to know) in its audience. It conveys an informing logic, a persuasive rhetoric, or a moving poetics that promises information and knowledge, insight and awareness" (Nichols 2001: 40). In Schamas Dokudrama wird dank des hohen Anteils an Fiktionalisierung das Bedürfnis nach Wissen bedient, und zwar in erheblichem Maße. Dies erfolgt jedoch gleichzeitig mit der Intention, Wissen populär und unterhaltend zu vermitteln, und zwar mit einer lebendigen und dramatischen Erzählung, mit einfühlbaren Figuren und mit einer visuellen Ästhetik, die über eine einfache Bebilderung weit hinausgeht.

Die Wichtigkeit der Fiktionalisierung bei der populären Geschichtsdarstellung, die sich wie andere populärkulturelle Produkte auch auf dem Medienmarkt bewähren muss, wird nicht zuletzt durch den Werbetext bestätigt, mit dem Schamas Dokudrama als DVD vermarktet wurde. Potenzielle Käufer werden auf dem Cover der DVD mit Aussagen wie den folgenden angesprochen:

The amazing story of the slaves who fought for Britain in the American War of Independence.

[...] In this fascinating film, Simon Schama tells the slaves' incredible story of escape and adventure $[\ldots]$.

Combining Simon Schama's compelling storytelling with reconstruction and dramatisation Rough Crossings tells the story [...].

Simon Schama brings to life the sentiments and sufferings of those caught up in this pivotal moment in history. It is a story charged with inspiration, terror, and, ultimately, 
betrayal that offers profound insights into contemporary life in Britain, America and Africa. (Rough Crossings 2007)

Narrativität, Dramatik und Emotionalität sind Elemente, die hier hervorgehoben werden und die vor allem mit fiktionalisierter Präsentation verbunden sind. Mit Ausnahme von Schama selbst scheint die Faktualität des Dokudramas von untergeordneter Attraktivität zu sein. Damit illustriert auch das Marketing von Rough Crossings einen Trend der Geschichtsdarstellung im Fernsehen, die sich seit ihren Anfängen immer stärker zu Hybridformen von fiktionalem und faktualem Erzählen entwickelt hat.

\section{Literatur}

Barnouw, Erik (1983) Documentary: A History of the Non-Fiction Film [1974]. 2. Aufl. Oxford: Oxford Univ. Press.

Bayer, Gerd (2008) „Fake Film as Media Criticism“. Anglistentag 2007, Proceedings. Hg. Klaus Stierstorfer. Trier: WVT. 143-64.

Brauburger, Stefan (2009) „Fiktionalität oder Fakten. Welche Zukunft hat die zeitgenössische Dokumentation?" History Goes Pop: Zur Repräsentation von Geschichte in populären Medien und Genres. Hgg. Barbara Korte und Sylvia Paletschek. Bielefeld: transcript. 203-13.

Cohn, Dorrit (1990) „Signposts of Fictionality. A Narratological Perspective“. Poetics Today 11.4: 775-804.

Downing, Taylor (2004) „Bringing the Past to the Small Screen“. History and the Media. Hg. David Cannadine. Basingstoke: Palgrave Macmillan. 7-19.

Fludernik, Monika (1996) Towards a ,Natural' Narratology. London: Routledge.

Hattendorf, Manfred (1995) „Fingierter Dokumentarfilm. Peter Delpeuts The Forbidden Quest (1993)“. Perspektiven des Dokumentarfilms. Hg. Manfred Hattendorf. München: diskurs film Verlag Schaudig \& Ledig. 191-211.

Heller, Heinz B. (1995) „Kulinarischer Dokumentarfilm? Anmerkungen zu neueren Tendenzen im Dokumentarfilm und seinem Verhältnis zur Unterhaltung“. Perspektiven des Dokumentarfilms. Hg. Manfred Hattendorf. München: diskurs film Verlag Schaudig \& Ledig. 97-110.

Hill, Annette (2005) Reality TV: Audiences and Popular Factual Television. London: Routledge.

Hohenberger, Eva (1998) „Dokumentarfilmtheorie. Ein historischer Überblick über Ansätze und Probleme“. Bilder des Wirklichen. Texte zur Theorie des Dokumentarfilms. Berlin: Vorwerk 8. 8-34.

Hohenberger, Eva, und Judith Keilbach (2003) „Die Gegenwart der Vergangenheit. Zum Verhältnis von Dokumentarfilm, Fernsehen und Geschichte“. Die Gegenwart der Vergangenheit: Dokumentarfilm, Fernsehen und Geschichte. Hgg. Eva Hohenberger und Judith Keilbach. Berlin: Vorwerk 8. 8-23. 
Izod, John, und Richard Kilborn (2000) Hgg. From Grierson to the Docu-Soap. Breaking the Boundaries. Luton: Univ. of Luton Press.

Jaeger, Stephan (2009) „Erzählen im historiographischen Diskurs“. Wirklichkeitserzäblungen: Felder, Formen und Funktionen nicht-literarischen Erzählens. Hgg. Christian Klein und Matías Martínez. Stuttgart: Metzler. 110-35.

Jost, François (1998) „Der Dokumentarfilm: Narratologische Ansätze“. Bilder des Wirklichen: Texte zur Theorie des Dokumentarfilms. Hg. Eva Hohenberger. Berlin: Vorwerk 8. 216-31.

Juhasz, Alexandra, und Jesse Lerner (2006) Hgg. F Is for Phony: Fake Documentary and Truth's Undoing. Minneapolis: Univ. of Minnesota Press.

Kilborn, Richard (2003) Staging the Real: Factual TV Programming in the Age of, Big Brother'. Manchester: Manchester Univ. Press.

Kilborn, Richard, und John Izod (1997) An Introduction to Television Documentary: Confronting Reality. Manchester: Manchester Univ. Press.

Klein, Christian, und Matías Martínez (2009) Hgg. Wirklichkeitserzählungen: Felder, Formen und Funktionen nicht-literarischen Erzäblens. Stuttgart: Metzler.

Korte, Barbara, und Sylvia Paletschek (2009) „Geschichte in populären Medien und Genres. Vom Historischen Roman zum Computerspiel“. History Goes Pop: Zur Repräsentation von Geschichte in populären Medien und Genres. Hgg. Barbara Korte und Sylvia Paletschek. Bielefeld: transcript. 9-60.

Korte, Barbara, und Eva Ulrike Pirker (2011) Black History - White History. Britain's Historical Programme between Windrush and Wilberforce. Bielefeld: transcript.

Leiser, Erwin (1992) „Dokumentarfilm und Geschichte“. Fernsebdokumentarismus. Bilanz und Perspektiven. Hg. Peter Zimmermann. München: Öhlschläger. 37-50.

Lipkin, Steven N. (2002) Hg. Real Emotional Logic: Film and Television Docudrama as Persuasive Practice. Carbondale: Southern Illinois Univ. Press.

Nichols, Bill (2001) Introduction to Documentary. Bloomington, IN: Indiana Univ. Press.

Paget, Derek (2011) No Other Way to Tell It: Dramadoc/Docudrama on Television [1998]. 2. Aufl. Manchester: Manchester Univ. Press.

Rhodes, Gary D., und John Parris Springer (2006) Hgg. Docufictions: Essays on the Intersection of Documentary and Fictional Filmmaking. London und Jefferson, NC: McFarland.

Roscoe, Jane, und Craig Haight (2001) Faking It: Mock-documentary and the Subversion of Factuality. Manchester: Manchester Univ. Press.

Rosenthal, Alan (1999) Hg. Why Docudrama? Fact-Fiction on Film and TV. Carbondale: Southern Illinois Univ. Press.

Condie, Steven (2007) Rough Crossings (Film). BBC und Thirteen/WNET New York.

Schama, Simon (2005) Rough Crossings: Britain, the Slaves and the American Revolution. London: BBC Books. 
Segeberg, Harro (2009) Hg. Referenzen: Zur Theorie und Geschichte des Realen in den Medien. Marburg: Schüren-Verlag.

Steinle, Matthias (2009) „Geschichte im Film. Zum Umgang mit den Zeichen der Vergangenheit im Dokudrama der Gegenwart“. History Goes Pop: Zur Repräsentation von Geschichte in populären Medien und Genres. Hgg. Barbara Korte und Sylvia Paletschek. Bielefeld: transcript. 147-65.

Viehoff, Reinhold (2002) „Gattung“. Metzler Lexikon Medientheorie / Medienwissenschaft: Ansätze - Personen - Grundbegriffe. Hg. Helmut Schanze. Stuttgart: Metzler. 125-27.

White, Hayden (1973) Metahistory. The Historical Imagination in Nineteenth-Century Europe. Baltimore: Johns Hopkins Univ. Press. 